\title{
Novel Calibration Technique for Wideband Transmitters using Constellation Mapping
}

\author{
Sidath Madhuwantha, Prasidh Ramabadran, Ronan Farrell and John Dooley \\ Maynooth University, Maynooth, Ireland
}

\begin{abstract}
Next generation wireless communication systems such as 5G will feature a step increase in the speed of wireless data transmission. This requires using RF signals of wider bandwidths along with higher order modulation schemes. Such signals are subjected to frequency and power dependent impairments such as gain compression, amplitude ripple and tilt, group-delay and AM-PM conversion as they traverse through the transmitter's hardware blocks. This paper introduces a novel calibration scheme that applies a specially designed wideband 64QAM modulated stimulus and uses the captured response to evaluate the impairments within the transmitter's RF chain. The needed pre-compensation is applied in a single iteration. This technique is intended for use with Doherty, Outphasing and MIMO type applications. Presented technique was experimentally validated using signal bandwidths up to $100 \mathrm{MHz}$ for both weakly and strongly nonlinear systems.

Index Terms - Wideband Vector Calibration.
\end{abstract}

\section{INTRODUCTION}

Next generation wireless communications such as $5 \mathrm{G}$ and High Throughput satellites (HTS) will feature a step increase in the data throughput to cater applications such as Ultra High Definition (UHD) media streaming and broadcasting, broadband internet connection speeds at gigabits per second and on demand gaming. Wireless channels with broader bandwidths are needed to support higher communication speeds. It has been identified previously that the limitations on the achievable wireless data throughput are primarily due to the physical impairments that Radio Frequency (RF) signals undergo while traversing through the RF hardware, and the propagation media owing to phenomena such as dispersion, frequency dependent gains and nonlinear transfer characteristics. Most transmitters employ parallel signal paths in quadrature relation to generate vector modulated waveforms for transmission where the amplitude and phase relations between the paths are critical to the integrity of the signal generated. One of the ways to mitigate the problem is to pre-compensate the signal of interest to minimize the impact of the impairments. This requires appropriate characterization of the impairments under the required operating conditions over the bandwidth of interest and generate the information required for calibration.

Calibration of an RF transmitter path has been extensively explored in the literature. In [1] RF path calibration employs a polar transmitter that generates a multi-sine signal stimulus. The response is measured in the time domain with a fourchannel digitizer consisting of four track-and-hold amplifiers and a high sampling oscilloscope. The cost of the test apparatus would be expensive at wider bandwidths due to the requirement of higher sampling rate equipment. In [2], a frequency swept technique is used where at least one spectral component is selected at a time and the best match is found iteratively. While in [3] single frequency sinusoids are employed and quadrature up-converted to a known RF. The up-conversion amplitude of the up-conversion image is measured and the amplitude and phase relations between the quadrature paths are tuned iteratively to minimize the image and this is repeated at multiple frequencies over the band of interest to gather the wideband calibration data. Techniques [2]-[3] are both iterative, the time required for calibration in fine steps will increase as the number of frequencies that need to be measured to cover the bandwidth of interest increases. Additionally, the waveforms used for calibration are unlike common wireless modulated signal standards.

We propose a non-iterative scheme that applies a specially designed 64-QAM modulated stimulus of scalable bandwidth to characterize the wideband impairments in a vector modulated transmitter. This modulation format is close to that of a standard modulation scheme used in practice. Its response in the band of interest is used to mitigate the impairments in the form of a correction filter. The remainder of the paper is structured as follows: In Section II the theoretical background of the proposed technique is given. Section III presents the experimentally measured results validating the proposed technique. Finally, the conclusions are presented.

\section{THEORETICAL BACKGROUND}

\section{A. Choice Of The Stimulus}

A 64-QAM modulated signal with a symbol rate of 80 mega-symbols per second with a raised cosine roll-off factor of 0.2 consists of multiple amplitudes and phases in its modulation constellation and sufficiently occupies the bandwidth of interest $(100 \mathrm{MHz})$ in the frequency domain. In theory, each transition between different symbols of a 64QAM constellation generates a different frequency component in the frequency domain. Therefore, a calibration signal with a well-defined constellation transmitted through a nonlinear system under test (SUT) can capture an accurate description of the frequency domain characteristics of the SUT within the bandwidth of interest. Accordingly, the stimulus was designed to contain all possible permutations of unique transitions of a 64-QAM constellation eq. (1). Moreover, stimulus s(t) having multiple amplitudes and phases excites not only frequency- 
dependent impairments, but also power-dependent impairments such as the AM-AM and AM-PM characteristics of the SUT. This helps in characterizing strongly nonlinear systems such as power amplifiers (PA) operating in the saturation region.

$$
{ }_{r}^{n} P=\frac{n !}{r !(n-r) !} \rightarrow{ }_{2}^{64} P=\frac{64 !}{(64-2) !}=4032
$$

The spectrum of the stimulus after applying QAM modulation, raised cosine filtering and hamming filtering for sharp band limiting is shown in Fig. 1. First, the stimulus signal is transmitted through a nonlinear SUT and the response $r(t)$ is captured using a reference receiver such as a vector signal analyzer (VSA) or a combination of a vector down-converter and an analog to digital converter (ADC).

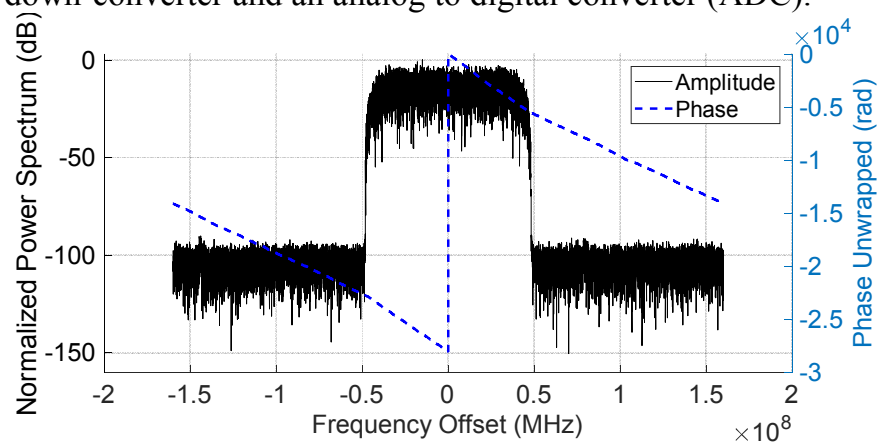

Fig. 1. Frequency domain spctra of magnitude and phase of stimuls.

The captured response $r(t)$ is then demodulated. Thereafter, the vector difference of the positioning of constellation symbols with respect to their ideal and captured location $\mathrm{c}(\mathrm{t})$ is calculated eq. (2). The digital correction factor needed for each of the constellation points to fall into their ideal location after transmission through SUT is represented by $\mathrm{c}(\mathrm{t})$, as indicated via Fig. 2.

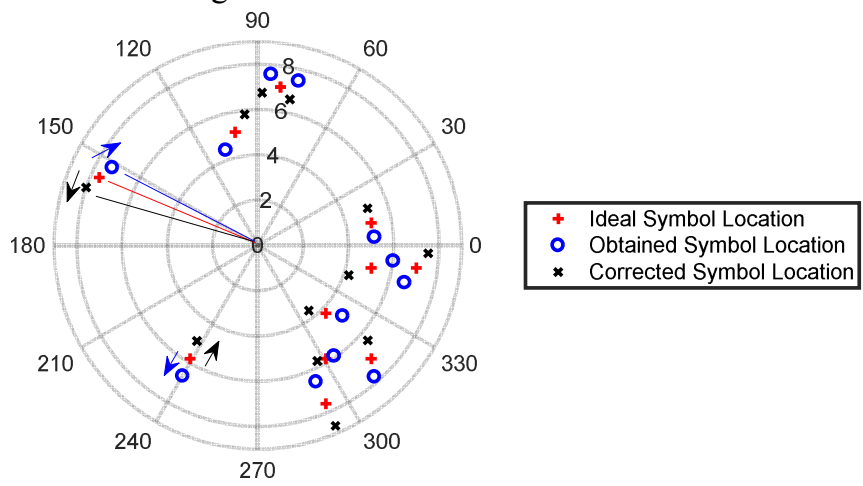

Fig. 2. Constellation is mapped on a polar plot with 64-QAM modulation symbols. Amplitude and phase variations can be captured and corrected. A 64-QAM modulated stimulus offers a higher level of amplitudes and phases in comparison to 16-QAM in time domain and a higher computational efficiency relative to a 256-QAM stimulus.

Next, a pre-compensated stimulus signal $\mathrm{p}(\mathrm{t})$ is generated via eq. (3). Afterwards, a finite impulse response filter (FIR) kernel encapsulating the transfer function of the SUT can be obtained using the deconvolution operation [6]. The coefficients of the FIR filter are represented by $f(n)$ in eq. (4). Finally, by applying the convolution operation eq. (5) between a desired signal $t_{x}(t)$ and the $f(n)$, it is possible to transmit information via the SUT with pre-compensation applied Tx(n) and this will yield a non-distorted output. The discrete domain representation of time domain signals is denoted by using $\mathrm{n}$ ( $\mathrm{n}$ $=1$ to 32768 , can be reduced depending on accuracy needed).

$$
\begin{gathered}
c(n)=s(n)-r(n) \\
p(n)=s(n)+c(n) \\
f(n)=\text { deconvolution }\{s(n), p(n)\} \\
\operatorname{Tx}(n)=\text { convolution }\{t x(n), f(n)\}
\end{gathered}
$$

\section{B. Specific Advantages Of The Stimulus Itself}

(a)

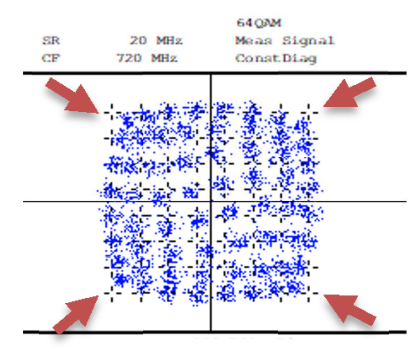

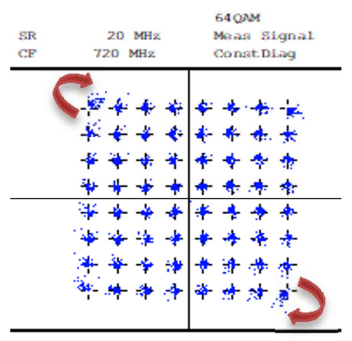

(b)
Fig. 3. VSA captures of (a) constellation of a strongly nonlinear system indicating outer constellation symbols subjected to AMAM distortion and inner symbols subjected to AMPM. (b) constellation of a weakly driven nonlinear system where outer symbols were subjected to AMPM conversion.

1. AMAM distortion identification - Constellation symbols are observed to have a shorter vector radii than their ideal locations as in Fig. 3 plot (a).

2. AMPM distortion identification - Results in the symbol point to have the same vector radius, but to have an angular movement along the vicinity of the ideal location. Observed in Fig. 3 plot (b).

3. Additionally, Inter-Symbol-Interference (ISI), random noise interference and phase noise can also be identified by observing the response of the stimulus [4].

\section{EXPERIMENTAL VALIDATION}

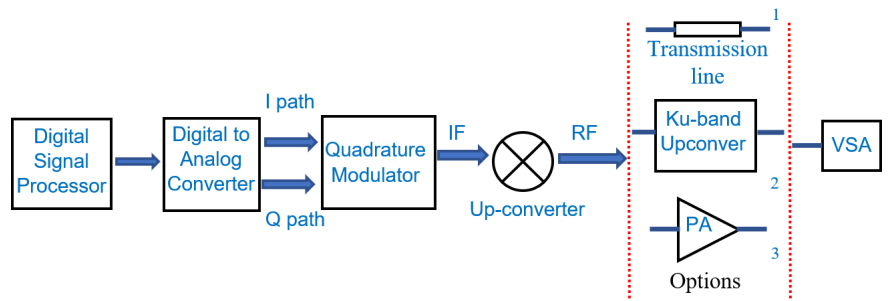

Fig. 4. Block level illustration of different RF transmit paths used. Option (1) S-band testing, (2) Ku-band testing and (3) strongly nonlinear system characterization using a Doherty Power Amplifier.

Comprehensive tests were performed to validate the robustness of the proposed technique at several frequencies, 
using three different test-steps, and with different input power levels. The tool MATLAB was used for the digital signal processing (DSP) operations [5]. Rohde \& Schwarz FSQ calibrated receiver of vector demodulation bandwidth of 120 $\mathrm{MHz}$ was used for acquisition of responses. The block diagram of the measurement apparatus is shown in Fig. 4. Options 1, 2 and 3 of were used to validate the test cases A, B, $\mathrm{C}$ and $\mathrm{D}$ as mentioned below. Test cases mentioned above were tested using the 64-QAM modulated stimulus itself as a common reference along with 8-PSK and different QAM modulation formats and signals for further validation.

\section{A. Weakly Nonlinear System Testing Of A Homodyne Modulator At S-band}

In addition to the stimulus signal (64-QAM) testing for this setup was also performed with an 8-PSK and 256-QAM modulated test signal. For 64-QAM, 8-PSK and 256-QAM test cases EVM readings of $1.788 \%, 1.881 \%$ and $1.907 \%$ were obtained at MER readings of $34.95 \mathrm{~dB}, 34.51 \mathrm{~dB}$ and $34.39 \mathrm{~dB}$ respectively. Constellation plots captured in S-band (at 2.32 GHz) for 8-PSK and 256-QAM are shown in Fig. 5.

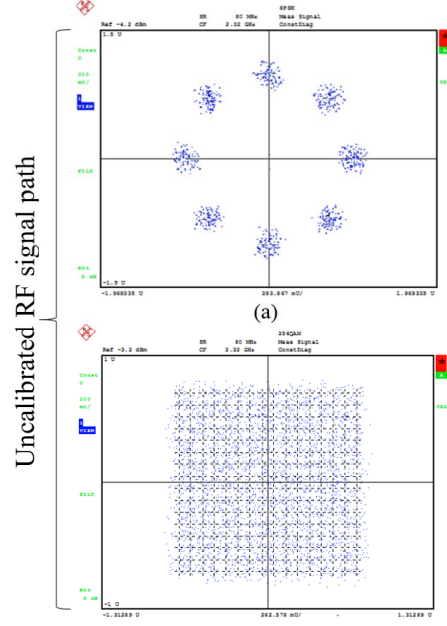

(c)

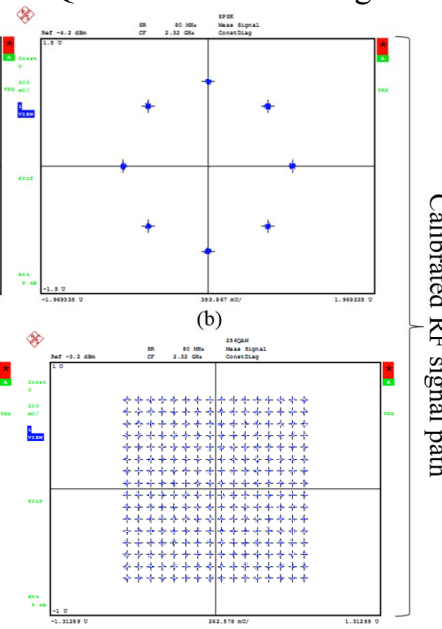

(d)
Fig. 5. VSA captures of demodulated 8-PSK and 256-QAM constellations $(\mathrm{a}, \mathrm{c})$ before and $(\mathrm{b}, \mathrm{d})$ after applying calibration.

\section{B. Weakly Nonlinear System Testing Of A Heterodyne Modulator At Ku-band}

Test bench in this case included a Ku-band up-converter with a cavity filter presenting high group-delay and higher amplitude roll-off over a bandwidth of $100 \mathrm{MHz}$.

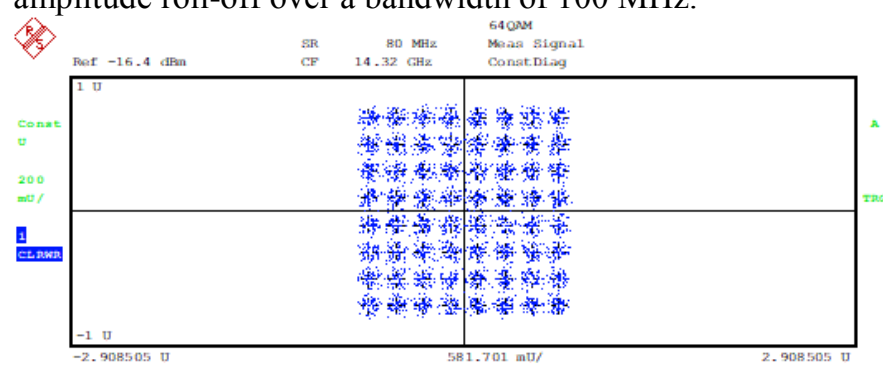

(a)

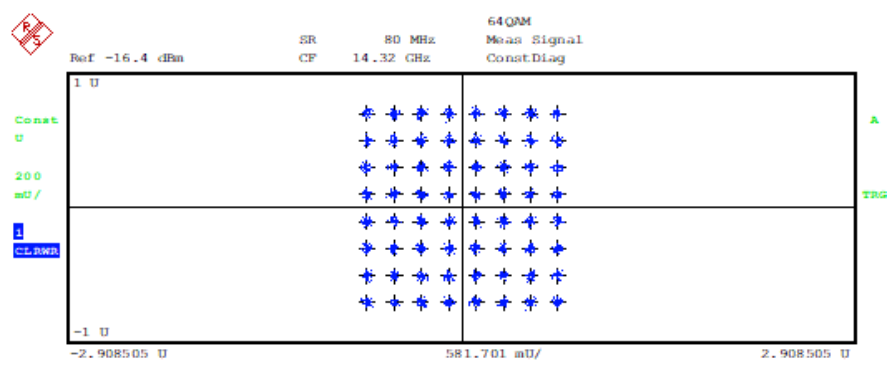

(b)

Fig. 6. Demodulated constellations of 64-QAM signals in Ku-band. (a) Uncalibrated, response and (b) calibrated response.

Similar to section (A), for 64-QAM, 8-PSK and 256-QAM test cases with bandwidth of $80 \mathrm{MHz}$ EVM readings of 3.401 $\%, 3.407 \%$ and $4.267 \%$ were obtained at MER readings of $29.37 \mathrm{~dB}, 29.35 \mathrm{~dB}$ and $27.40 \mathrm{~dB}$ respectively.

\section{Weakly Nonlinear System with a Doherty Power Amplifier}

The system was assembled as indicated in option (3) of Fig. 4. The Doherty power amplifier (DPA) was driven by a wideband $(80 \mathrm{MHz}) 64-\mathrm{QAM}$ modulated signal inputs with a low input power, resulting in a weakly nonlinear system. In addition to the calibration signal, ten different 64-QAM modulated signals were generated and used to prove the consistency of the method. As explained in section II, each transition in the 64-QAM constellation corresponds to a unique frequency component in the frequency domain. Testing with ten different 64-QAM signals with random transitions in their constellations allows to further validate the process of designing of the constellation of the stimulus signal to have all possible transitions between its 64 constellation points (1).

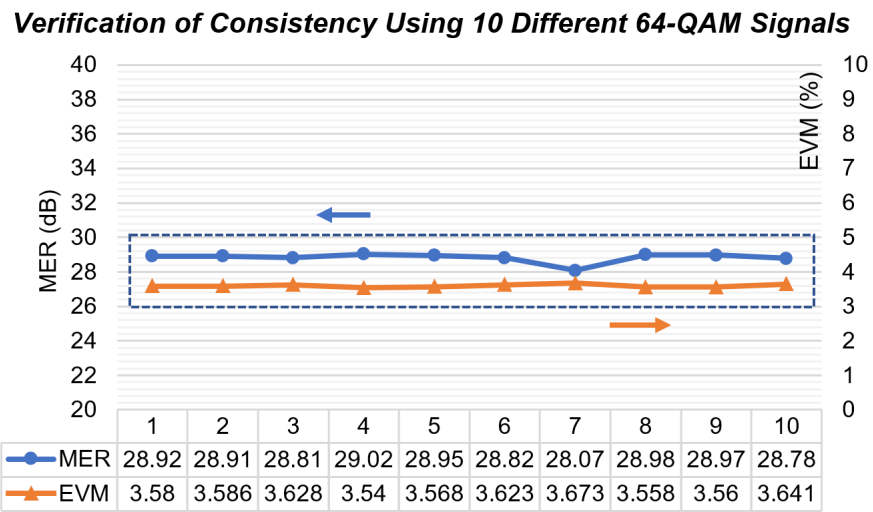

Fig. 7. Demodulated results for EVM and MER performance of ten different 64-QAM signals after performing calibration $\{1-10\}$.

Following Fig. 7, a flat response for MER and EVM was obtained from ten different 64-QAM test cases as depicted by test results from 1 to 10 (MER was within $1.4 \mathrm{~dB}$ of variation and EVM results were within $<1 \%$ of deviation). Additionally, for an 8-PSK modulated test signal an EVM of $3.55 \%$ and a MER of $28.99 \mathrm{~dB}$ was obtained. This was an improvement of MER by $12.33 \mathrm{~dB}$ and EVM by $11.14 \%$ in comparison to the uncalibrated case and results are on par with performance 
observed for different 64-QAM modulated signals. Fig. 8 indicates the improvements achieved in the spectral responses.

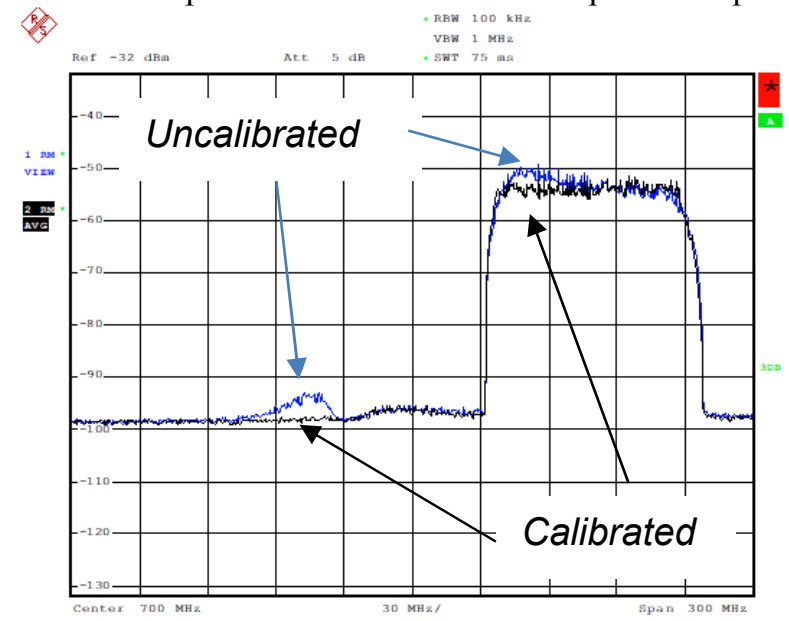

Fig. 8. Capture of the magnitude spectrum of a 64-QAM signal with and without calibration applied. Compensation of amplitude rolloff and image rejection can be observed.

\section{Strongly Nonlinear System with a Doherty Power Amplifier}

Following option (3) in Fig. 4, a strongly nonlinear system is in operation when the DPA is driven with sufficient input power to operate beyond $\mathrm{P} 1 \mathrm{~dB}$ compression point. Due to the strong nonlinearity both power and frequency dependent nonlinearities are in effect. Power dependent impairments functioning as AM-AM and AM-PM conversion effects that are nonexistent/not-apparent (Fig. 6. (a)) in weakly nonlinear systems exists in a strongly nonlinear system (Fig. 10. (a).) and pose a special challenge for RF path calibration. The knowledge of these impairments is essential to accomplish path calibration and digital pre-distortion (DPD) of PAs.

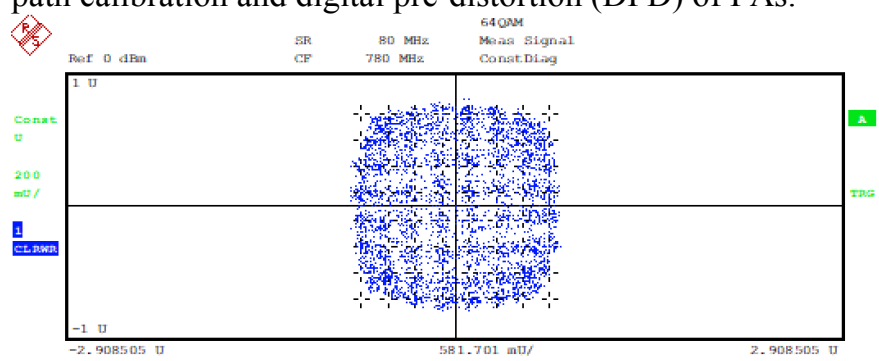

(a)

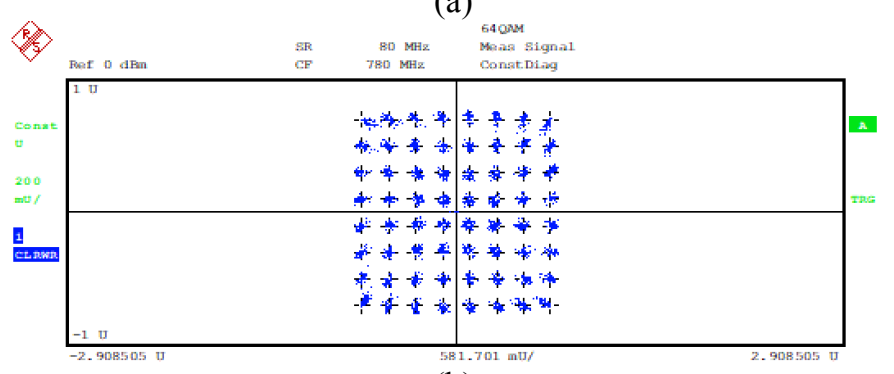

(b)

Fig. 9. Constellation view of VSA captures obtained for strongly nonlinear test-setup driven with $80 \mathrm{MHz}$ wideband (a) uncalibrated (EVM of $12.144 \%$, MER $18.31 \mathrm{~dB}$ ), (b) calibrated (EVM 5.509\%, MER 25.18 dB) 64-QAM signal.
The challenge is significant in a DPA as the RF signal must propagate through two amplifiers biased at different classes (Class AB, Class $\mathrm{C}$ ) and then be constructively recombined. The demodulated responses of Fig. 10 (a) indicate significant AM-AM, AM-PM distortions with ISI. Post-calibration not only frequency dependent impairments were compensated but also power dependent impairments as observed in Fig. 10. (b).

Moreover, significant reduction in ISI was achieved postcalibration with improvements in EVM by $6.64 \%$ and in MER by $6.87 \mathrm{~dB}$. After performing calibration, indicated linear performance is as if the system is driven at a lower input power level, delivering higher output power without heavy distortion in the RF signal constellation and reduced MER.

\section{CONCLUSION}

A novel method based on vector positioning of constellation symbols of a specially designed 64-QAM modulated signal that can be used to identify RF path impairments was proposed with experimental validation and repeated measurements to characterize both weakly and strongly nonlinear systems. Advantages of the technique includes; use of a modulated waveform that closely resembles a current standard communication signal format, the ability of the chosen stimulus to alleviate both frequency and power dependent impairments such as amplitude rippletilt, group-delay, AM-AM and AM-PM conversion. These advantages were proven by a variety of experimental test cases at different frequency bands and at different power levels using three different test-setups.

\section{ACKNOWLEDGEMENT}

This publication has emanated from research conducted with the financial support of Science Foundation Ireland (SFI) and is co-funded under the European Regional Development Fund under Grant Number 13/RC/2077.

\section{REFERENCES}

[1] K. El-Akhdar, S. Ahmed, G. Neveux, D. Barataud and J. M. Nebus, "Calibrated RF time-domain measurement of non-linear devices in digital polar transmitter architecture," 2013 European Microwave Conference, Nuremberg, 2013, pp. 452-455.

[2] H. Shi, H. Jensen, "Calibrating an RF transmitter," U.S. Patent US20050059361A1, 2005.

[3] A Khandelwal, A Verma, "A Novel Gain, Phase and Offset Calibration Scheme for Wideband Direct-Conversion Transmitters", 2015 IEEE 81st Vehicular Technology Conference, Glasgow, 2015, pp. 1-5.

[4] Keysight Technologies, Appl. Note, "Predicting Residual BER II: Measurements for Lowering Radio Cost".

[5] J. van der Geest, "permn $(\mathrm{V}, \mathrm{N}, \mathrm{K})$ - File Exchange - MATLAB Central." MathWorks File Exchange, 2016.

[6] P. Ramabadran et al., "Digitally Assisted Wideband Compensation of Parallel RF Signal Paths in a Transmitter," 2018 91st ARFTG Microwave Measurement Conference, Philadelphia, PA, 2018, pp. 1-4. 